\title{
Fluxonic cellular automata
}

\author{
M. V. Miloševića) \\ Departement Fysica, Universiteit Antwerpen, Groenenborgerlaan 171, B-2020 Antwerpen, Belgium \\ and Department of Physics, University of Bath, Claverton Down, Bath BA2 7AY, United Kingdom \\ G. R. Berdiyorov and F. M. Peeters ${ }^{\text {b) }}$ \\ Departement Fysica, Universiteit Antwerpen, Groenenborgerlaan 171, B-2020 Antwerpen, Belgium
}

(Received 25 July 2007; accepted 5 September 2007; published online 19 November 2007)

\begin{abstract}
We propose a quantum cellular automata composed of nanostructured mesoscopic superconducting squares, where the logic states are defined by two trapped vortices in a $2 \times 2$ blind hole matrix. We present the functioning logic gate based on this fluxonic cellular automata, where the logic operations are verified through theoretical simulations within the Ginzburg-Landau formalism. The input signals are defined using the vortex interaction with current loops placed on top of the two diagonal blind holes of the input cell. The readout technology may be chosen from a large variety of modern vortex imaging methods, transport, and local density of states measurements. (C) 2007 American Institute of Physics. [DOI: 10.1063/1.2813047]
\end{abstract}

Quantum dot cellular automata (QCA), first described a decade ago by Lent et al., ${ }^{1}$ is one of the proposed nanotechnologies that may replace traditional complementary metaloxide semiconductor scaling in microelectronics. However, despite having been physically demonstrated at a small scale, the attractiveness of QCA begins to diminish as logic complexity grows. As a novel device design, superconducting nanostructured arrays in a magnetic field have been recently considered for storing information. ${ }^{2}$ When a magnetic field is applied to a superconductor, the flux enters in the form of individual quantized vortices which repel each other. In contrast to many other systems, the density of vortices, as well as the strength and range of their interaction, can be easily tuned by an external field, temperature, or geometry of the sample. ${ }^{3}$ This sets the ground for the novel field of fluxtronics based on the controlled motion of flux quanta. ${ }^{4}$ In addition, if needed, individual vortices can be trapped by magnetic nanodots ${ }^{5}$ or by antidots perforating the sample. ${ }^{6}$ Such combined properties make superconductors ideal for designing micromagnetic flux quanta machines. ${ }^{7}$

In this letter, we explore the idea of creating a superconducting analogue of a QCA. As the unit cell, we use a mesoscopic superconducting square, with a regular $2 \times 2$ array of blind holes (see Fig. 1). Unlike antidots, blind holes have a thin superconducting bottom layer, which allows the trapped flux to remain as separated single quantum vortices inside the pinning sites. ${ }^{8}$ If specific conditions are met, two vortices nucleate in the sample, and their position, as compared to the single electron charges in QCA, determines the logic state. As we will show, this state can be toggled externally, as well as by magnetic fields emerging from the adjacent cells.

To show the basics of this fluxonic cellular automata (FCA), we consider an Al square with side $w=3.0 \mu \mathrm{m}$ and thickness $d=150 \mathrm{~nm}$, containing four blind holes with side $w_{h}=850 \mathrm{~nm}$ and bottom thickness $d_{h}=30 \mathrm{~nm}$, and an interdistance between the holes of $w_{0}=350 \mathrm{~nm}$. The operating temperature is taken $T=0.94 T_{c}$. The coherence length $\xi(0)$

\footnotetext{
${ }^{a)}$ Electronic mail: milorad.milosevic@ua.ac.be

${ }^{b)}$ Electronic mail: francois.peeter@ua.ac.be
}

$=120 \mathrm{~nm}$ and the penetration depth $\lambda(0)=140 \mathrm{~nm}$ were estimated experimentally for similar $\mathrm{Al}$ samples in Ref. 9. Since the chosen parameters are compatible with standard lithography, the unit cell described here is experimentally realizable, though smaller devices would be needed for potential applications.

Within the time-dependent Ginzburg-Landau (TDGL) theory, we investigated the superconducting state of the latter sample when exposed to a homogeneous external magnetic field $H_{\text {ext }}$. In search for the steady-state solution of two coupled GL equations for the order of parameter and the vector potential, we compare the energies of all found stable states (for details, see Ref. 10). The complete Gibbs free energy $(\mathcal{G})$ diagram, as a function of magnetic field is shown in Fig. 2(a). With increasing $H_{\text {ext }}$, the ground state goes through vortex configurations denoted by successive vorticity $L$, up to the critical field $H_{c 3}=4.352 \mathrm{mT}$. Note that the energy barrier between consecutive states is much larger than the thermal activation energy $k_{B} T_{c}$, and thermal noise driven transitions are not possible (though closer to $T_{c}$ saddle-point energies decrease). As emphasized in the inset of Fig. 2(a), vortex states exhibit enhanced stability for even vorticity. This feature results from the commensurability effects between the vortices and the pattern of blind holes. ${ }^{6,9}$ $|\psi|^{2}$-density contourplots in Figs. 2(b)-2(e) show the vortex configurations for up to four captured vortices.
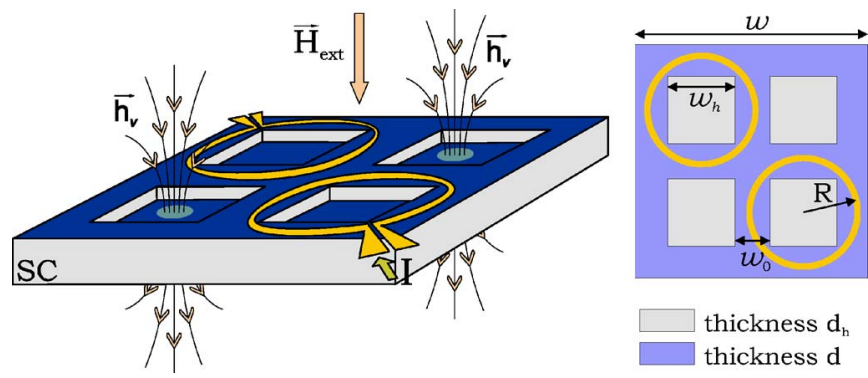

FIG. 1. (Color online) Scheme of the fluxonic cellular automata unit cell: a superconducting square with $2 \times 2$ blind holes and current loops on top in an external magnetic field $H_{\text {ext }}$. Magnetic field lines $h_{\nu}$ illustrate induced vortices in the sample. 

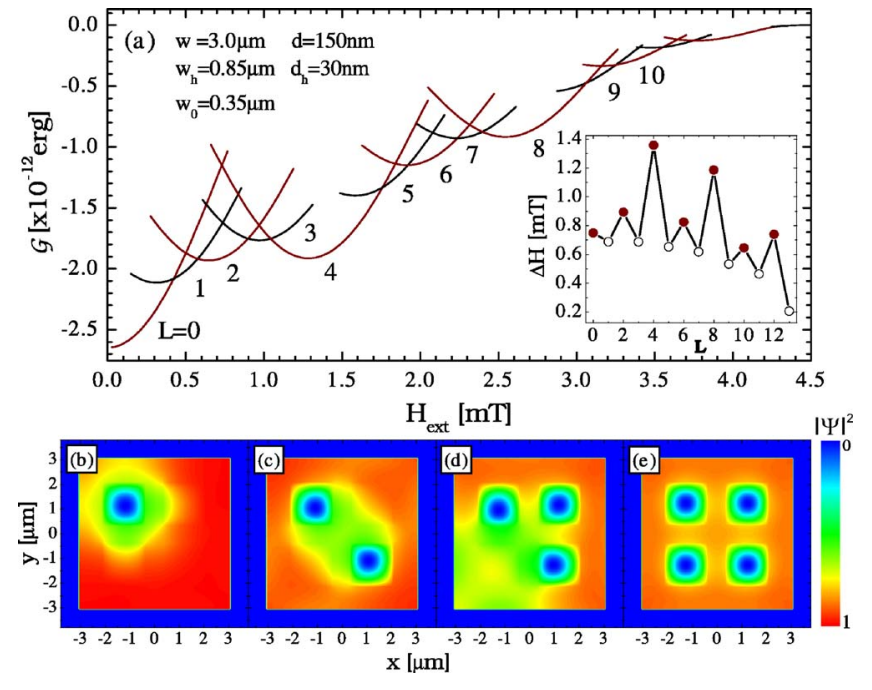

FIG. 2. (Color online) (a) The Gibbs free energy as a function of the applied magnetic field $H_{\text {ext }}$ for the allowed superconducting states with different vorticities $L$. The inset shows the stability field range for each vortex state. [(b)-(e)] The equilibrium position of the vortices is illustrated by the Cooper-pair density plots for states $L=1-4$, respectively.

The vortex state that we are particularly interested in is the $L=2$ state, which is found stable in the field range $H_{\text {ext }}=0.2746-1.1905 \mathrm{mT}(0.575-0.824 \mathrm{mT}$ as the ground state). When two external flux lines are trapped in the sample, due to their mutual repulsion, they can occupy the blind holes along either one of the diagonals. We use these two energetically degenerate states to represent a logic value 1 and a logic value 0 . However, in order for this FCA unit cell to meet all criteria for performing computations, one must be able to uniquely predefine the logic state. To secure a well-defined input for the FCA, we propose placing submicron current-carrying loops on top of the two diagonal blind holes, as depicted in Fig. 1. We aim to use the magnetic properties of the current loops, which, when the created magnetic moment is parallel to the external field, attract the vortices and vice versa. ${ }^{5}$ Therefore, to change the FCA input state, we simply change the polarity of the applied currents in the loops.

To remain within the current experimental limitations, we present results for the current loops with radius $R=600 \mathrm{~nm}$, and assume them to be electronically decoupled from the superconductor by a $50 \mathrm{~nm}$ oxide layer. Using TDGL, we investigate the time evolution of the superconducting state in response to the applied current $I$. In our iteration procedure, each step is related to a predefined fraction of the GL time $\tau_{\mathrm{GL}}=4 \pi \sigma_{n} \lambda(T)^{2} / c^{2}$, where $\sigma_{n}$ is the normal-state conductivity (for mesoscopic Al samples we obtained $\tau_{\mathrm{GL}} \approx 2.8 \mathrm{ps}$ at $T=0.94 T_{c}$ ). The results of our simulation are presented in Fig. 3, as the time relaxation of the superconducting condensate when forced to transit between the two degenerate $L=2$ states at $H_{\text {ext }}=0.645 \mathrm{mT}$. In Fig. 3, the cell is initially in state 1 . In order to change the logic state to 0 , we apply clockwise loop currents $I=0.32 \mathrm{~mA}$ which attract vortices.

As illustrated by Cooper-pair density insets, both transitions occur via the diagonals of the sample, where the barrier between the states is lowest. One should note that if currents remain on (dotted lines in Fig. 3), the 0 and 1 state do not have equal energies, due to the influence of the stray field of the current loops on the sample. For 0 and 1 state to remain Downloaded 20 Nov 2007 to 143.129.131.61. Redistribution subject

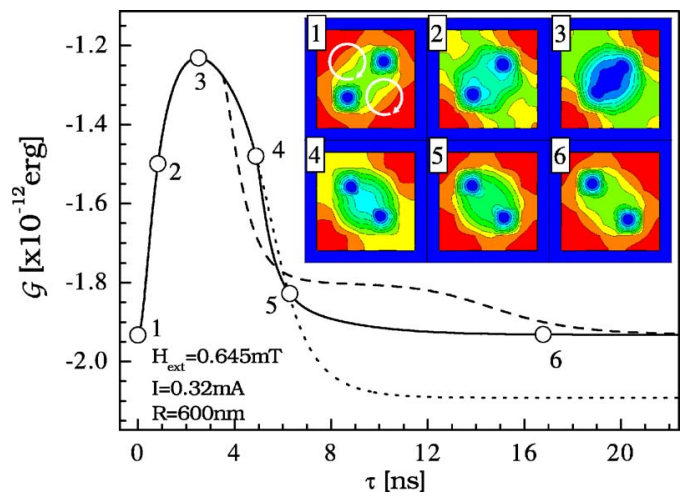

FIG. 3. (Color online) Evolution of the Gibbs free energy during the $1 \rightarrow 0$ transition. The insets show snapshots of the Cooper-pair density profiles at intervals indicated by open dots in the energy curve. The current was switched off after $\Delta \tau=4.5$ (dashed line) and $5.5 \mathrm{~ns}$ (solid line), or not at all (dotted line).

degenerate after the switching, the current must be turned off after the energy barrier is crossed, as shown by the dashed and solid lines in Fig. 3. In that case, the system needs somewhat longer time to equilibrate. Nevertheless, switching time is still very fast, and remains under $20 \mathrm{~ns}$ (we neglect the time for stabilizing the current in the loop $t_{\mathrm{ON}} \sim \rho C$ ). Our simulation is also valid for high- $T_{c}$ superconductors, where $\tau_{\mathrm{GL}}$ (and consequently switching times) may be order of magnitude smaller than in $\mathrm{Al}$ due to poor conductivity.

Of course, the value of the current necessary for switching between the logic states may not be chosen freely. In one limit, the applied current could be insufficient to make the vortices overcome the $0 \rightarrow 1(1 \rightarrow 0)$ energy barrier. On the other hand, large currents could either over-repel the vortices and expel them from the sample or become sufficiently strong to create an additional vortex in the system. In any case, the logic state would be returnlessly lost. Figure 4 shows these threshold currents as a function of the applied magnetic field.

In an isolated cell, the two logic states have identical energy and, hence, are occupied with equal probability. However, with more cells in the immediate vicinity, the energetically favorable state is determined by the total magnetic energy of the vortex fields emerging from all cells. As a result, if one cell is fixed at a particular state, a linear chain of cells [an information "wire" (Ref. 1)] will all assume the

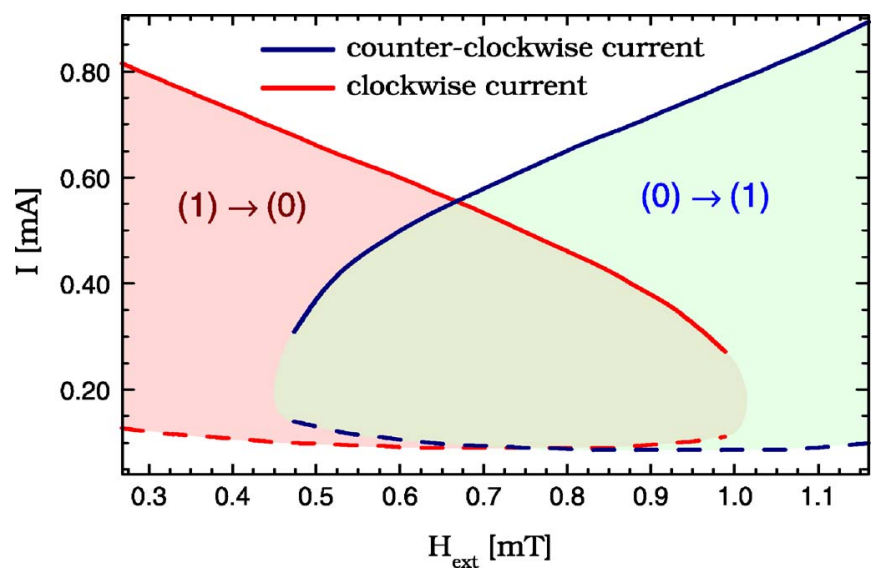

FIG. 4. (Color online) Operating window for the current through the loops to induce transitions between the two logic states. The dashed (solid) curves indicate the lower (upper) threshold current.

to AIP license or copyright; see http://apl.aip.org/apl/copyright.jsp 


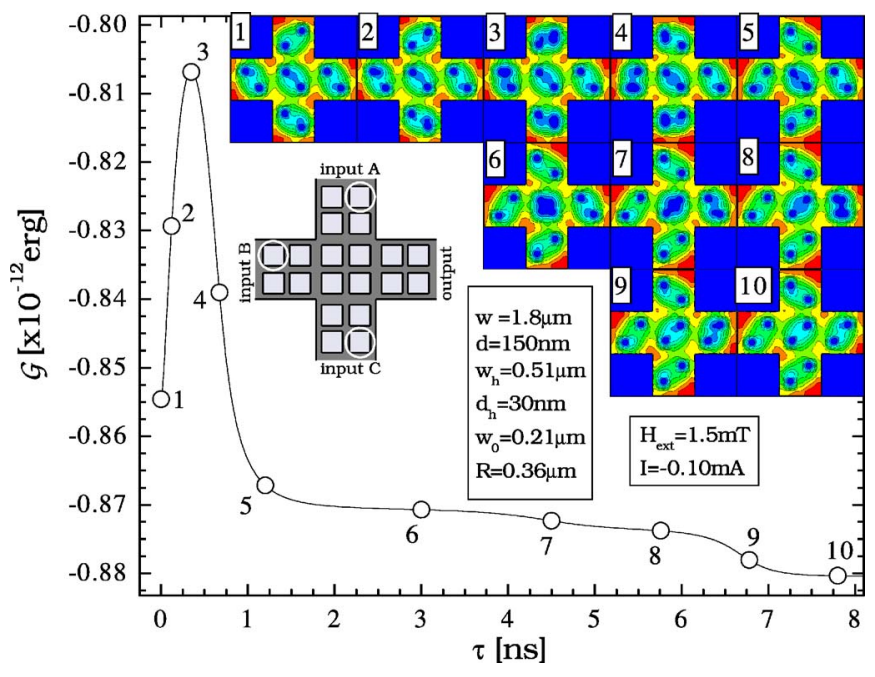

FIG. 5. (Color online) The operation process of the majority gate is illustrated by the Cooper-pair density insets corresponding to the superconducting states denoted by open dots in the free energy curve. Current loops are shown by white circles in the lower inset and define the input as $A=0$, $\mathrm{B}=1$, and $\mathrm{C}=1$.

same logic state to minimize the interaction energy. However, very different from the standard QCA case, in the FCA the interaction between the adjacent cells may be strengthened by conjoining the edges of the cells. Thus, the whole gate may be carved out of a singly connected sample, in which case vortices do not interact solely via fields but also through local supercurrents.

One of the fundamental logic devices is a three-input majority logic gate. In a FCA realization shown in Fig. 5, this gate consists of five unit cells: a central logic cell, three inputs labeled A, B, and C, and an output cell. Inputs determine the state of the central cell, which can assume either polarization, while the output polarization follows that of the central cell. The majority gate actually performs the Boolean function: out $=\mathrm{AB}+\mathrm{BC}+\mathrm{AC}$, and can be programmed to act as an OR gate or an AND gate by fixing one input to 1 or 0 , respectively. As an example, we demonstrate the operation of the gate when having inputs $\mathrm{A}=0, \mathrm{~B}=1$, and $\mathrm{C}=1$. While keeping the rest same as above, here we reduced the lateral dimensions of each cell by $40 \%$ (i.e., $w=1.8 \mu \mathrm{m}$, $w_{h}=510 \mathrm{~nm}, w_{0}=210 \mathrm{~nm}$, and $R=360 \mathrm{~nm}$ ) to improve the switching times. In order to have two vortices in each cell in the ground state, we take the applied field $H_{\text {ext }}=1.5 \mathrm{mT}$. To further illustrate rich possibilities, the logic state in the input cells is defined by only one current loop. While the physics involved remains the same, this simplifies the experimental realization of the device. These loops are placed in such a way to ensure $\mathrm{A}=0, \mathrm{~B}=1$, and $\mathrm{C}=1$ for the same value and direction of the current in all of them $(I=-0.10 \mathrm{~mA})$. In addition to the well-defined output illustrated in Fig. 5 by the snapshots of the Cooper-pair density during the transitions, one should notice that the total operation time is multiply decreased by the reduction of the unit-cell size. This time, including the switching process for five cells, is below $10 \mathrm{~ns}$ and is faster than the single switching demonstrated in Fig. 3, even though only one current loop was used (and consequently the time necessary to overcome the energy barrier is larger).

To summarize, we introduced a fluxonic concept for cellular automata, where two flux lines in the superconducting square with $2 \times 2$ blind holes perform the logic operations. Adjacent unit cells may be spatially connected, which enhances interactions and decreases the switching time, while in standard QCA such realization is not possible. All unit cells in the FCA are experiencing the same conditions (applied field and temperature) and therefore the circuits may have large number of cells without endangering the data transfer. By further improving the QCA concept, feedback is easily prevented in the FCA by current loops, which can serve either to fix the input signal or to remember a particular state in transfer cells during the computation. Previously realized QCA based on Si quantum dots operate at very low temperatures $(\sim 100 \mathrm{mK})$, since the electrostatic interaction energy must be significantly larger than the thermal one, while the FCA circuits can operate close to the critical temperature of the used superconductor (for cuprates, $T_{c}$ $\sim 90 \mathrm{~K})$. We demonstrated FCA operation frequencies in the gigahertz range, even for low- $T_{c}$ microscale samples, while showing that smaller scales, higher temperatures, and optimization of the sample geometry significantly decrease these operating times. Various vortex-imaging methods or a recently developed multiple-tunnel-junction method $^{11}$ can serve as very accessible readout techniques for the FCA.

This work was supported by the Flemish Science Foundation (FWO-Vl), the Belgian Science Policy, and the ESFAQDJJ network. M.V.M. acknowledges support from EU Marie-Curie Intra-European Program. G.R.B. acknowledges support from FWO-Vlaanderen.

${ }^{1}$ C. S. Lent, P. D. Tougaw, W. Porod, and G. H. Bernstein, Nanotechnology 4, 49 (1993)

${ }^{2}$ M. B. Hastings, C. J. Olson Reichhardt, and C. Reichhardt, Phys. Rev. Lett. 90, 247004 (2003).

${ }^{3}$ A. K. Geim, I. V. Grigorieva, S. V. Dubonos, J. G. S. Lok, J. C. Maan, A. E. Filippov, and F. M. Peeters, Nature (London) 390, 256 (1997).

${ }^{4}$ F. Nori, Science 271, 1373 (1996).

${ }^{5}$ For review, see, I. F. Luyksutov and V. L. Pokrovsky, Adv. Phys. 54, 67 (2005).

${ }^{6}$ M. Baert, V. V. Metlushko, R. Jonckheere, V. V. Moshchalkov, and Y. Bruynseraede, Phys. Rev. Lett. 74, 3269 (1995).

${ }^{7}$ S. Savel'ev and F. Nori, Nat. Mater. 1, 179 (2002); J. E. Villegas, S. Savel'ev, F. Nori, E. M. Gonzalez, J. V. Anguita, R. Garcia, and J. L. Vicent, Science 302, 1188 (2003); D. Cole, S. Bending, S. Savel'ev, A. Grigorenko, T. Tamegai, and F. Nori, Nat. Mater. 5, 305 (2006); C. C. de Souza Silva, J. Van de Vondel, M. Morelle, and V. V. Moshchalkov, Nature (London) 440, 651 (2006).

${ }^{8}$ A. Bezryadin, A. Buzdin, and P. Pannetier, Phys. Rev. B 53, 8553 (1995).

${ }^{9}$ T. Puig, E. Rosseel, M. Baert, M. J. Van Bael, V. V. Moshchalkov, and Y. Bruynseraede, Appl. Phys. Lett. 70, 3155 (1997); G. R. Berdiyorov, B. J. Baelus, M. V. Milošević, and F. M. Peeters, Phys. Rev. B 68, 174521 (2003).

${ }^{10}$ M. V. Milošević and F. M. Peeters, Phys. Rev. Lett. 94, 227001 (2005).

${ }^{11}$ A. Kanda, B. J. Baelus, F. M. Peeters, K. Kadowaki, and Y. Ootuka, Phys. Rev. Lett. 93, 257002 (2004). 